\title{
Monitoramento e nível de ingestão de desoxinivalenol por trigo
}

\section{Monitoring and ingestion of deoxynivalenol by wheat}

\author{
Joice Sifuentes dos Santos $^{1 *}$; Tatiane Martins de Oliveira ${ }^{1}$; Ligia Manoel Martins ${ }^{2}$; \\ Elisabete Hiromi Hashimoto ${ }^{3}$; Manoel Carlos Bassoi ${ }^{4}$; João Leonardo Fernandes \\ Pires $^{5}$; Martha Zavariz Miranda $;$; Sandra Garcia ${ }^{6}$; Eiko Nakagawa Itano ${ }^{7}$; Elisabete \\ Yurie Sataque Ono ${ }^{8}$; Osamu Kawamura ${ }^{9}$; Elisa Yoko Hirooka ${ }^{6}$
}

\section{Resumo}

O trigo é um dos alimentos mais consumidos na dieta humana, contribuindo com cerca de $20 \%$ das calorias diárias. Considerando a predominância de Fusarium graminearum neste grão, a contaminação por desoxinivalenol (DON) foi avaliada em trigo dos Estados do Paraná (21 amostras) e do Rio Grande do Sul (15 amostras), assim como se estimou o risco da sua ingestão diária no município de LondrinaPR, Brasil. Utilizando Cromatografia Líquida de Alta Eficiência (CLAE), DON foi detectado em 72,2 $\%$ das amostras, variando de não detectável a 1592,21 $\mu \mathrm{g} / \mathrm{kg}$, nível médio $321,59 \mu \mathrm{g} / \mathrm{kg}$ (limite de quantificação $=87,75 \mu \mathrm{g} / \mathrm{kg}$ ). Para calcular a Ingestão Diária Estimada (IDE) de DON foi aplicado Questionário de Freqüência de Consumo de Alimentos a 260 indivíduos (8-76 anos, 64,8 \% do gênero feminino e $35,2 \%$ do masculino). Os derivados mais consumidos foram massas alimentícias ( $151 \mathrm{~g} /$ semana) e pão francês ( $267 \mathrm{~g} / \mathrm{semana})$. Baseado nesses alimentos, a IDE média de DON foi de 0,308 $\mu \mathrm{g} / \mathrm{kg}$ peso corporal $/$ dia, sendo que dois indivíduos $(0,8 \%)$ apresentaram IDE superior a Ingestão Diária Máxima Tolerável Provisória (IDMTP) recomendada de $1 \mu \mathrm{g} / \mathrm{kg}$ p.c./dia. A importância de contínuo monitoramento perante perigo de exposição a DON é evidente, sendo que os níveis máximos em trigo no Brasil foram recentemente publicados pela ANVISA.

Palavras-chave: Consumo de trigo, exposição estimada, micotoxinas

\begin{abstract}
Wheat comprises one of the main ingredients in human diet, accounting for approximately $20 \%$ of daily calories. Concerning the predominant frequency of deoxynivalenol (DON) producing Fusarium graminearum in wheat, DON occurrence in the State of Paraná (21 samples) and Rio Grande do Sul (15 samples), as well as the risk assessment of daily intake of DON in Londrina-PR, Brazil were evaluated. DON was detected in $72.2 \%$ samples by high performance liquid chromatography (HPLC), which ranged from non-detectable to $1,592.21 \mu \mathrm{g} / \mathrm{kg}$, with average of $321.59 \mu \mathrm{g} / \mathrm{kg}$ (limit of quantification= $87.75 \mu \mathrm{g} /$
\end{abstract}

\footnotetext{
1 Programa de Pós-Graduação em Ciência de Alimentos. Universidade Estadual de Londrina, UEL. E-mail: joice.sifuentes@ gmail.com; tatianeoliveira_z@hotmail.com

2 Graduanda do Curso de Farmácia. Universidade Estadual de Londrina, UEL. E-mail: lili_profecia@yahoo.com.br

3 Prof ${ }^{a}$ da Universidade Federal Tecnológica do Paraná, Campus de Francisco Beltrão. E-mail: hi_bete@yahoo.com.br

4 Pesquisador da EMBRAPA. Centro Nacional de Pesquisa da Soja, Londrina, PR. E-mail: bassoi@cnpso.embrapa.br

5 Pesquisador da EMBRAPA. Centro Nacional de Pesquisa do Trigo, Passo Fundo, RS. E-mail: pires@cnpt.embrapa.br; marthaz@ cnpt.embrapa.br

${ }^{6}$ Prof $^{\mathrm{a}} \mathrm{s}$ do Dept $^{\mathrm{o}}$ de Ciência e Tecnologia de Alimentos, Universidade Estadual de Londrina, UEL. E-mail: sgarcia@uel.br; hirooka@uel.br

7 Prof $f^{a}$ do Depto de Ciências Patológicas, Universidade Estadual de Londrina, UEL. E-mail: itanoeiko@hotmail.com

8 Prof $f^{a}$ do Depto de Bioquímica e Biotecnologia, Universidade Estadual de Londrina, UEL. E-mail: eysono@hotmail.com

9 Prof. do Food Hygiene Laboratory, Kagawa University. E-mail: kawamura@ag.kagawa-u.ac.jp

* Autor para correspondência
} 
$\mathrm{kg}$ ). In order to calculate the estimated daily intake (EDI) of DON, a Food Frequency Questionnaire was applied to 260 persons ( $64.8 \%$ female and $35.2 \%$ male, from 8 to 76 years-old). The main food ingested were pasta (151 g/week) and bread (267 g/week), and based in these items, the average estimated daily intake (EDI) of DON was $0.308 \mu \mathrm{g} / \mathrm{kg}$ body weight/day. This EDI was lower than Provisional Tolerable Daily Maximum Intake (PTDMI) settled in $1 \mu \mathrm{g} / \mathrm{kg}$ b.w./day, although two persons showed estimated DON ingestion above PTDMI. The data ratify the importance of continuous monitoring and risk assessment targeted on DON exposure.

Key words: Wheat intake, estimated exposure, mycotoxins

\section{Introdução}

O trigo (Triticum aestivum L.) ocupa $30 \%$ da área mundial cultivada com cereais, com produção anual aproximada de 500 milhões de toneladas (EMBRAPA, 2010). A produção nacional de trigo atingiu 6 milhões de toneladas na safra 2008, caindo para apenas 4,9 milhões de toneladas em 2009, devido as condições climáticas. A região Sul é a principal produtora de trigo do país, sendo 53 \% produzido pelo Estado do Paraná, seguido pelo Rio Grande do Sul (30 \%). No entanto, o consumo interno de 10 milhões de toneladas ainda requer importação do produto (IBGE, 2009). Este cereal contribui com cerca de $20 \%$ das calorias diárias ingeridas, além de fornecer proteínas de elevado valor biológico e carboidratos complexos (amido e fibras), bem como vitaminas do complexo B e ferro.

Fusarium graminearum Schwabe (teleomorfo Giberella zeae Shw.) causa giberela ou fusariose, doença de ampla ocorrência em trigo, cuja produção de desoxinivalenol (DON ou vomitoxina) durante o cultivo no campo depende das condições metereológicas, práticas culturais e susceptibilidade da cultivar, entre outros. DON é tricoteceno do grupo $\mathrm{B}$, que além de dupla ligação nas posições $\mathrm{C}_{9,10}$ e anel epóxido na posição 12,13 , caracterizase por apresentar carbonila no $\mathrm{C}_{8}$. $\mathrm{A}$ exposição a níveis elevados de DON pode desencadear vômito, perda de peso, dores abdominais, diarréia e imunossupressão. DON é classificado pela International Agency for Research on Cancer no Grupo 3, isto é, não carcinogênico para humanos (IARC, 1993).
A literatura aponta diversas patologias

relacionadas ao consumo de cereais contaminados por toxinas de Fusarium spp., desde alimentary toxic aleukia (ATA) na Rússia (STEYN, 1995) a puberdade precoce, câncer cervical e hiperplasias (BRIONES-REYES; GOMÉZ-MARTINEZ; CUERVA-ROLÓN, 2007). No Brasil, os escassos trabalhos limitam-se à ocorrência de DON em trigo e derivados (OLIVEIRA et al., 2002; LAMARDO; NAVAS; SABINO, 2006; CALORI-DOMINGUES et al., 2007). Salienta-se que DON tem sido a toxina de Fusarium spp. mais freqüentemente reportada no mundo, assim como detectada em maior concentração, sendo considerada como "indicadora" de contaminação por este fitopatógeno (PRANGE et al., 2005).

Micotoxinas constituem toxinas naturais de ocorrência inevitável em alimentos, apontada pela avaliação de risco (risk assessment) como perigo crônico na dieta, somando-se a contaminantes sintéticos, aditivos alimentares e resíduos de agrotóxicos. A avaliação de risco e a exposição estimada são importantes ferramentas para avaliar os riscos de micotoxinas na cadeia alimentar a humanos e a animais, devido ao efeito dependente da dose consumida, toxicidade do composto, peso corporal e condição física do indivíduo, além da presença de outras micotoxinas e fator dietético, capazes de aumentar ou diminuir a toxicidade (KUIPER-GOODMAN, 1988).

No presente trabalho procedeu-se avaliação preliminar de DON em trigo com enfoque aos dois principais Estados produtores, Paraná e Rio Grande do Sul, bem como se estimou a exposição a DON por meio do consumo de derivados de trigo em amostra 
populacional do município de Londrina-PR.

\section{Material e Métodos}

\section{Amostragem}

Um total de 36 amostras de trigo em grão (Triticum aestivum L.) foi coletado nas regiões Norte (Londrina e Sertanópolis, entre 23ํ19' e $23^{\circ} 50$ de Latitude Sul e entre $21^{\circ} 22^{\prime}$ e $51^{\circ} 30^{\prime}$ de Longitude Oeste) e Sudoeste do Paraná (Dois Vizinhos, 254ㄴ S e $53^{\circ} 03^{\prime}$ O), além de Norte/Noroeste (Passo Fundo e Três de Maio, entre $27^{\circ} 46^{\prime}$ e 28 $30^{\circ}$ 'O e entre $54^{\circ} 14^{\prime}$ e $50^{\circ} 53^{\prime}$ O) e Nordeste (Vacaria, 28 $30^{\circ}$ 'S e $\left.50^{\circ} 56^{\prime} \mathrm{O}\right)$ do Rio Grande do Sul. As 15 amostras do Norte Paranaense pertenceram a safras 2006 (sete amostras), 2007 (quatro) e 2008 (quatro amostras), cultivadas em campos experimentais da Empresa Brasileira de Pesquisa Agropecuária, em Londrina (EMBRAPA Soja, 11 amostras) e fornecidas por Empresa processadora de trigo de Sertanópolis (quatro amostras). Seis amostras recém-colhidas no Sudoeste do Paraná (safra 2008) foram adquiridas numa Cooperativa armazenadora de grãos. As amostras do Rio Grande do Sul consistiram de sete amostras cultivadas na região Norte/Noroeste e oito na região Nordeste (safras 2006, 2007 e 2008, quatro, sete e quatro amostras), pertencentes a campos experimentais da EMBRAPA Trigo e parceiros da Unidade. A amostra (500 g) foi quarteada e, $100 \mathrm{~g}$ trituradas na granulometria de 20 mesh, acondicionadas em frasco plástico e mantidas a $-20{ }^{\circ} \mathrm{C}$ até a análise. As contra-amostras foram armazenadas a $4{ }^{\circ} \mathrm{C}$.

\section{Análise de desoxinivalenol}

DON com $98 \%$ de pureza (Sigma, St Louis, Estados Unidos) foi suspenso em acetato de etila e quantificado a $260 \mathrm{~nm}$, empregando absortividade molar de 1410 (UV-VIS Cintra 20, GMB, Melborne, Austrália), conforme preconizado pelo Instituto Adolfo Lutz (IAL, 2008).

Quinze gramas de amostra foram adicionadas de
$3 \mathrm{~g}$ de $\mathrm{NaCl}$ e $120 \mathrm{~mL}$ de água ultrapura (Purelab Elga, Lane End, Inglaterra), agitadas a 150 r.p.m. por 30 min (Marconi, Piracicaba, Brasil) e filtrada em filtro de papel, seguida de microfibra (GF/C poro 1,2 $\mu \mathrm{m}$, Whatman, Maistone, Inglaterra). Coluna de imunoafinidade (Dontest ${ }^{\circledR}$, Vicam, Watertown, Estados Unidos) foi utilizada para pré-limpeza de 2 $\mathrm{mL}$ do extrato, lavada com $20 \mathrm{~mL}$ de água ultrapura, seca e o eluato obtido com $2 \mathrm{~mL}$ de metanol (grau HPLC, J. T. Baker, Xalostoc, México) e seco sob fluxo de $\mathrm{N}_{2}$ a $40{ }^{\circ} \mathrm{C}$. A película foi ressuspensa em $300 \mu \mathrm{L}$ de fase móvel (metanol:água, 30:70, v/v) e $20 \mu \mathrm{L}$ analisados em duplicata em cromatógrafo líquido de alta eficiência (LC-10AD, Shimadzu, Kyoto, Japão) utilizando coluna nucleosil 100 C18 (5 $\mu \mathrm{m} ; 250$ x 4 mm; Merck, Darmstadt, Alemanha) sob fluxo de $0,7 \mathrm{~mL} / \mathrm{min}$ e detector de arranjo de fotodiodo (PDA) na faixa entre 190 e $400 \mathrm{~nm}$ (SPD10A UV/VIS, Shimadzu, Kyoto, Japão). DON apresentou máxima absorvância em $220 \mathrm{~nm}$.

A curva de calibração foi confeccionada nas concentrações de 250, 500, 750, 1000 e 1500 ng/ $\mathrm{mL}$ em metanol:água $(30: 70, \mathrm{v} / \mathrm{v})$, correspondente a níveis entre 300 e $1800 \mu \mathrm{g} / \mathrm{kg}$ de DON em trigo. Trigo artificialmente contaminado com 250, 500, 750 e $1000 \mu \mathrm{g} / \mathrm{kg}$ de DON apresentou recuperação média de 75,4\% (faixa de 53,1 a 118,7\%). Os limites de detecção $(\mathrm{LD}=0,28 \mu \mathrm{g} / \mathrm{kg}$, correspondente a 3 x Desvio Padrão) e quantificação ( $\mathrm{LQ}=87,75$ $\mu \mathrm{g} / \mathrm{kg}, 10 \mathrm{xDP}$ ) foram calculados baseados em branco da amostra. Valores inferiores ao LQ foram considerados como zero e incluídos nos cálculos.

Todos os procedimentos envolvidos foram conduzidos conforme protocolo de segurança e descontaminação descrito para análise de micotoxinas (IAL, 2008).

\section{Análise bromatológica}

A composição centesimal, determinada em porcentagem, consistiu de umidade, proteínas, lipídios e resíduos minerais em base seca ( $\mathrm{g} \%$ ), conforme descrito em Normas do Instituto Adolfo 
Lutz (IAL, 2008). A porcentagem de carboidratos em base seca ( $\mathrm{g} \%$ ) foi obtida por diferença (100 g - gramas totais de umidade, proteína, lipídios e cinzas).

\section{Avaliação da freqüência do consumo de produtos a base de trigo}

O consumo de produtos a base de trigo no município de Londrina-PR foi avaliado aplicando Questionário de Freqüência de Consumo de Alimentos (QFCA) a 260 indivíduos (SANTOS et al., 2009). Além de informação sobre o consumo, também foram coletados dados como sexo, peso e altura. A quantidade ingerida foi estimada baseada na porção alimentar por medida caseira para cada produto (BRASIL, 2003) e freqüência de consumo ("não consumo", "eventualmente", "cada 15 dias", "1-2 vezes/semana", "3-4 vezes/semana", "5-6 vezes/semana", “1 vez/dia" e "2 vezes/dia”).

\section{Exposição estimada a DON}

O cálculo de Ingestão Diária Estimada (IDE) requer informação perante contaminação e consumo do alimento. A contaminação por DON em grãos de trigo, obtida no presente monitoramento, foi aliada ao fator de processamento e associados aos resultados do QFCA, que estimou o consumo local de produtos à base de trigo. Valores de DON em trigo abaixo do limite de quantificação (LQ) foram assumidos como $1 / 2$ LQ para o cálculo de IDE (IPCS/GEMS, 1995). Considerando a quantificação de DON em grãos de trigo, para o cálculo de provável nível em farinha utilizou-se o fator de processamento de $70 \%$ (extração de farinha a partir de trigo in natura). Nesse valor obtido, para panificação, assumiu-se adição de $60 \%$ de peso devido à absorção de água e outros ingredientes, isto é, $100 \mathrm{~g}$ de grão transformado em $112 \mathrm{~g}$ de pão. Para a produção de massas alimentícias assumiu-se o valor de $30 \%$ de peso adicional, ou seja, $100 \mathrm{~g}$ de grão transformado em $91 \mathrm{~g}$ de massa alimentícia. A
IDE individual foi calculada excluindo os indivíduos que não informaram peso, bem como dados sobre quantidade consumida, segundo a fórmula:

$\operatorname{IDE}(\mu \mathrm{g} / \mathrm{kg}$ peso corporal $/$ dia $)=$

DON em trigo $(\mu \mathrm{g} / \mathrm{kg})$ x consumo do alimento $(\mathrm{kg} / \mathrm{dia})$

peso do indivíduo $(\mathrm{kg})$

\section{Análise estatística}

Os valores da composição centesimal de trigo nos Estados produtores foram analisados pelo teste de normalidade de Liliefors (AYRES et al., 2007) e não apresentaram distribuição normal, portanto, aplicou-se o teste não paramétrico de Mann-Whitney. Os dados de DON nas regiões dos dois Estados, assim como a Ingestão Diária Estimada de DON obtida a partir de pão francês e massas alimentícias também não apresentaram distribuição normal, portanto, aplicou-se o teste não paramétrico de Kruskal-Wallis. Considerando que as diferenças dos níveis de DON entre as safras não foram estatisticamente significativas, a análise foi realizada por região sem considerar o número de amostras de cada safra.

As análises foram realizadas utilizando o programa Bioestat 5.0 (Belém, Pará, Brasil), considerando nível de $5 \%$ de probabilidade.

\section{Resultados e Discussão}

Aumidade nas amostras de trigo analisadas variou entre 10,5 e 14,2 g \% (Tabela 1), com oito amostras apresentando nível superior a $13 \%$, recomendado para conservação e empacotamento (BRASIL, 2001). Os teores de cinzas, proteínas, extrato etéreo e carboidratos apresentaram-se consistentes com dados da literatura (FUJITA; FIGUEROA, 2003). O teor de cinzas do trigo paranaense apresentouse superior ao do riograndense $(p<0,05)$, podendo a diferença ser atribuída a fatores como cultivar, dureza do grão, condições climáticas e solo (Tabela 1). 
Tabela 1. Composição bromatológica de trigo ( $n=36$ amostras) cultivado nos Estados do Paraná e Rio Grande do Sul (safras 2006, 2007 e 2008).

Composição

$(\mathrm{g} \% *)$
Localidade

\begin{tabular}{lcllc}
\hline \multicolumn{2}{c}{ Paraná $(\mathbf{n = 2 1})$} & & \multicolumn{2}{c}{ Rio Grande do Sul $(\mathbf{n}=\mathbf{1 5})$} \\
\cline { 1 - 2 } \cline { 5 - 5 } Média & Faixa & & Média & Faixa \\
$12,51^{\mathrm{a}}$ & $10,50-14,20$ & & $12,86^{\mathrm{a}}$ & $11,40-14,20$ \\
$1,37^{\mathrm{a}}$ & $1,17-1,74$ & & $1,18^{\mathrm{b}}$ & $0,80-1,56$ \\
$11,43^{\mathrm{a}}$ & $5,22-15,55$ & & $10,91^{\mathrm{a}}$ & $8,95-12,20$ \\
$1,31^{\mathrm{a}}$ & $0,75-2,16$ & & $1,35^{\mathrm{a}}$ & $0,92-2,31$ \\
$73,38^{\mathrm{a}}$ & $66,79-80,99$ & & $73,70^{\mathrm{a}}$ & $71,26-75,71$ \\
\cline { 5 - 6 } & & & &
\end{tabular}

* em base seca.

Letras diferentes na mesma linha indicam diferença significativa segundo o teste de Mann-Whitney $(\mathrm{p}<0,05)$.

Com relação a concentração de DON, dez amostras $(27,8 \%)$ exibiram contaminação inferior ao limite de quantificação $(87,75 \mu \mathrm{g} / \mathrm{kg}$ ) (Tabela 2). Houve diferença nos níveis de DON entre os dois Estados, sendo $163,03 \mu \mathrm{g} / \mathrm{kg}$ o nível médio de DON nas amostras do Paraná e 543,57 $\mu \mathrm{g} / \mathrm{kg}$ do Rio Grande do Sul $(p<0,05)$. As amostras de trigo provenientes da região Nordeste do Rio Grande do Sul apresentaram contaminação média de $741,83 \mu \mathrm{g} / \mathrm{kg}$, enquanto da região Norte/Noroeste apresentou 316,99 $\mu \mathrm{g} / \mathrm{kg}$, superior aos valores detectados na região Norte $(195,11$ $\mu \mathrm{g} / \mathrm{kg})$ e Sudoeste $(90,35 \mu \mathrm{g} / \mathrm{kg})$ do Paraná $(\mathrm{p}<0,05)$. Embora as seis amostras do Sudoeste do Paraná tenham sido recém-colhidas (safra 2008) e as 15 amostras do Norte Paranaense em 2006, 2007 e 2008, a diferença no tempo de armazenagem das amostras não geraria mudanças no nível de toxina entre o tempo inicial de armazenagem e o momento de análise das amostras. A produção de micotoxinas por Fusarium graminearum e outras espécies do gênero Fusarium é pouco provável durante o armazenamento, especialmente em grãos com umidade inferior a $14 \%$ e quando as condições de armazenamento são adequadas. Ramírez, Chulze e Magan (2006) observaram que não ocorreu produção de DON por duas cepas de $F$. graminearum em grãos de trigo armazenados a $5^{\circ} \mathrm{C}$ durante 49 dias de incubação.
Os autores também não observaram produção de DON em trigo com atividade de água entre 0,90 e 0,93. Estima-se que trigo com 14,5\% de umidade tenha uma atividade de água de 0,766 (RAMIREZ; CHULZE, MAGAN, 2006). Ono et al. (2002) avaliaram a microbiota fúngica e os níveis de fumonisinas em milho recém-colhido e após 12 meses de armazenagem (11 e $14 \%$ de umidade). Os autores relataram que a contagem de Fusarium spp. permaneceu inalterada durante os 12 meses de armazenamento do cereal. $\mathrm{O}$ mesmo foi observado para o conteúdo de fumonisinas, não havendo diferença significativa em milho recémcolhido e após 12 meses.

Salienta-se a importância da precipitação pluviométrica e temperatura como fatores determinantes no nível de micotoxinas produzidas no campo, favorecendo a multiplicação de $F$. graminearum e conseqüente produção de fusariotoxinas. Durante o plantio da safra 2006 na região Norte/Noroeste do Rio Grande do Sul, a precipitação variou de $37 \mathrm{~mm}$ (maio) a $167 \mathrm{~mm}$ (junho) e cerca de $100 \mathrm{~mm}$ na colheita (outubro-novembro), em contraste a maior umidade na safra 2007 (68 mm em junho, $325 \mathrm{~mm}$ em julho e 300 $\mathrm{mm}$ na colheita). A safra 2008 apresentou precipitação de $59 \mathrm{~mm}$ em julho, atingindo $350 \mathrm{~mm}$ em outubro. 
$\mathrm{Na}$ região Norte Paranaense, a precipitação da safra 2006 variou de $15 \mathrm{~mm}$ (agosto) e $160 \mathrm{~mm}$ na colheita (setembro), em relação a safra 2007 com 4 $\mathrm{mm}$ (setembro) a $223 \mathrm{~mm}$ (julho) e safra $2008 \mathrm{com}$ $24 \mathrm{~mm}$ (julho) a $185 \mathrm{~mm}$ (agosto). As amostras da safra 2008 do Sudoeste Paranaense expuseram à precipitação de $50 \mathrm{~mm}$ (julho) a $300 \mathrm{~mm}$ (outubro). A Figura 1 apresenta a precipitação pluviométrica e temperatura média mensais no campo para os anos 2006, 2007 e 2008, nos Estados estudados.

Figura 1. Precipitação pluviométrica $(\mathrm{mm})$ e temperatura média $\left({ }^{\circ} \mathrm{C}\right)$ mensais nas regiões Norte/Noroeste e Nordeste do Rio Grande do Sul e Norte e Sudoeste do Paraná nas safras 2006, 2007 e 2008, no período em que o trigo estava no campo (maio a outubro/novembro - Rio Grande do Sul, abril a setembro - Paraná). Os dados referentes às temperaturas da região Nordeste do Rio Grande do Sul não foram disponibilizados.
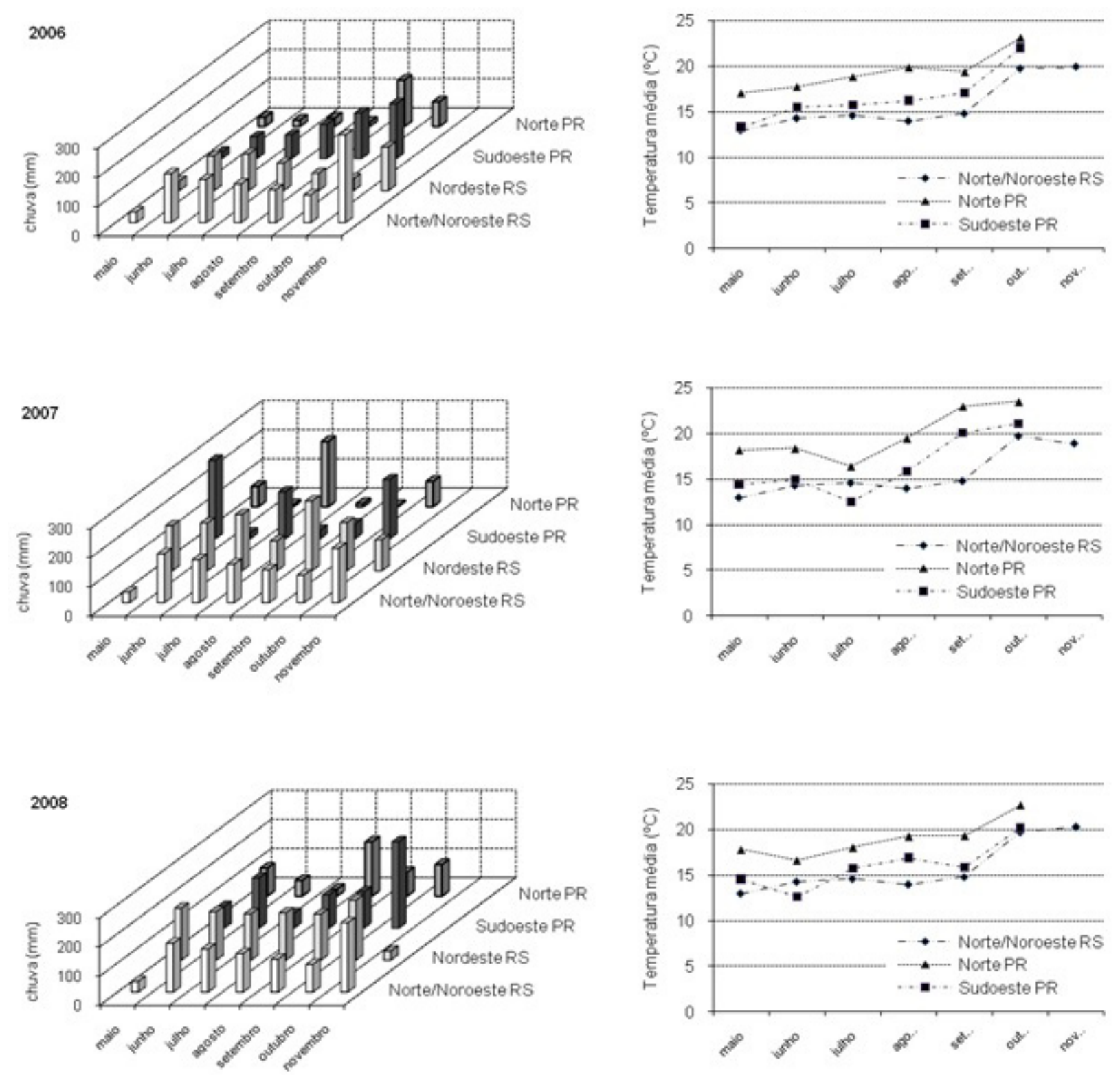

Fonte: Dados climatológicos - IAPAR; Centro de Meteorologia Aplicada - FEPAGRO. 
Observou-se que $72,2 \%$ das 36 amostras de trigo analisadas apresentaram contaminação por DON (> LQ), com níveis variando de 89,45 a $1592,21 \mu \mathrm{g} / \mathrm{kg}$ (amostras positivas). A contaminação de DON em 95,2 \% do trigo cultivado no Paraná situou-se entre não detectado e $500 \mu \mathrm{g} / \mathrm{kg}$, enquanto aproximadamente $35 \%$ das amostras do Rio Grande do Sul apresentaramse entre 750,1 e $1750 \mu \mathrm{g} / \mathrm{kg}$ (Figura 2). Esses valores foram inferiores ao limite máximo estabelecido pela Comunidade Européia para cereais não-processados, de $1750 \mu \mathrm{g} / \mathrm{kg}$ para trigo e aveia (COMMISSION OF THE EUROPEAN COMMUNITIES, 2007). A ANVISA publicou em fevereiro deste ano a nova legislação sobre os limites máximos de micotoxinas em alimentos. Está previsto para aplicação a partir de janeiro de 2012 o limite de $2000 \mu \mathrm{g} / \mathrm{kg}$ de DON em trigo integral, farinha de trigo, trigo para quibe e farelo de trigo (BRASIL, 2011). Na Legislação preconizada pela Commission of the European
Communities (2007), além de máximo permitido em cereais não transformados, existem valores máximos para farinha de cereais $(750 \mu \mathrm{g} / \mathrm{kg})$, pães, produtos de pastelaria, bolachas, refeições leves à base de cereais e cereais para refeições $(500 \mu \mathrm{g} / \mathrm{kg})$, massas alimentícias secas $(750 \mu \mathrm{g} /$ $\mathrm{kg}$ ) e alimentos destinados a lactantes e crianças $(200 \mu \mathrm{g} / \mathrm{kg})$.

Ao analisar os resultados de composição bromatológica (Tabela 1) e níveis de DON em trigo (Tabela 2), não se observou correlação entre estes parâmetros $(p>0,05)$. Ono et al. (2006) também não observaram correlação dos níveis de fumonisinas com conteúdos protéico e lipídico de milho paranaense. Todavia, Vieira, BadialeFurlong e Oliveira (1999) procedendo análise de contrastes, obtiveram correlação entre umidade e cinzas com níveis de aflatoxina, ocratoxina e zearalenona em farinha de trigo do Rio Grande do Sul.

Figura 2. Distribuição dos níveis de DON em trigo cultivado ( $n=36$ amostras) nos Estados do Paraná (PR) e Rio Grande do Sul (RS) das safras de 2006, 2007 e 2008.

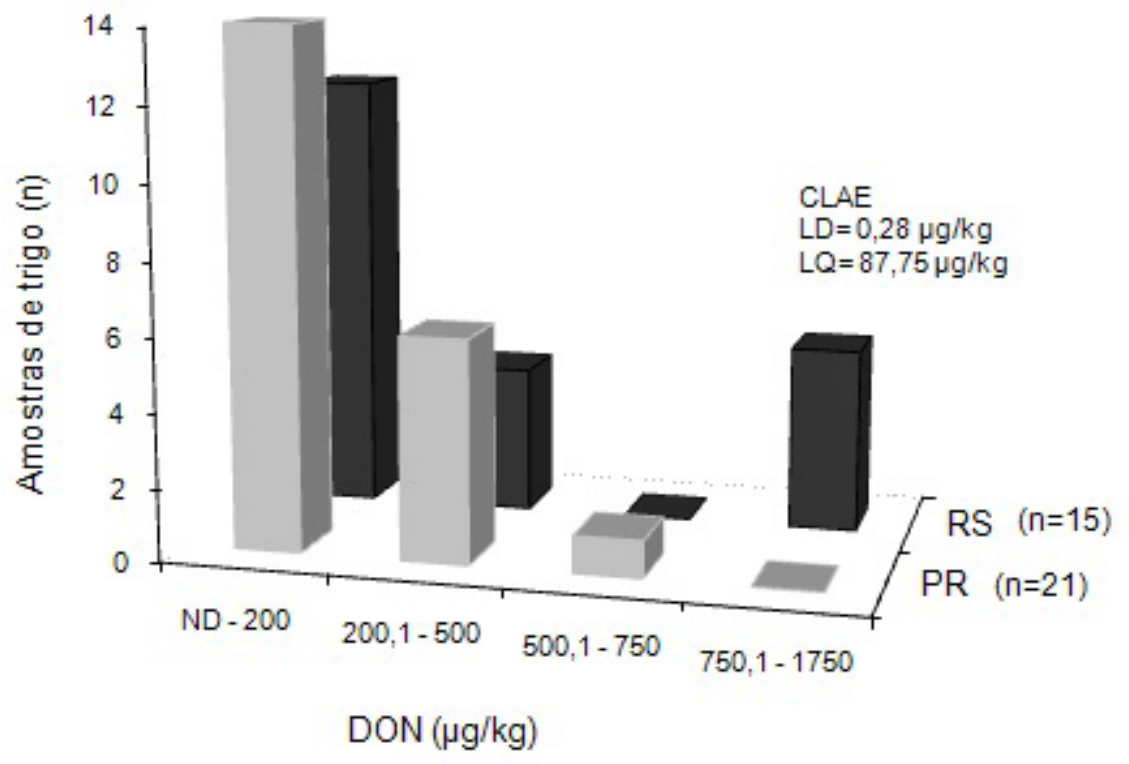


Tabela 2. Níveis de desoxinivalenol (DON) em grãos de trigo ( $\mathrm{n}=36$ amostras) cultivado nos Estados do Paraná e Rio Grande do Sul (safras 2006, 2007 e 2008).

\begin{tabular}{|c|c|c|c|c|c|c|}
\hline \multirow{3}{*}{ Localidade } & \multicolumn{6}{|c|}{ DON ( $\mu \mathrm{g} / \mathbf{k g})$} \\
\hline & \multirow[t]{2}{*}{ Região } & \multicolumn{2}{|c|}{ Total } & \multicolumn{3}{|c|}{ Amostras positivas } \\
\hline & & $\mathbf{N}$ & Média & $\mathbf{N}(\%)$ & Média & Faixa \\
\hline \multirow[t]{3}{*}{ Paraná } & Norte & 15 & $195,11^{\mathrm{a}, \mathrm{b}}$ & $10(66,6)$ & 288,17 & $112,76-609,62$ \\
\hline & Sudoeste & 6 & $90,35^{\mathrm{b}}$ & $4(66,6)$ & 135,52 & $92,90-200,29$ \\
\hline & Total & 21 & 163,03 & $14(66,6)$ & 244,55 & $92,90-609,62$ \\
\hline \multirow[t]{3}{*}{ Rio Grande do Sul } & Norte/Noroeste & 7 & $316,99^{\mathrm{a}, \mathrm{b}}$ & $4(57,1)$ & 554,74 & $89,45-1592,21$ \\
\hline & Nordeste & 8 & $741,83^{a}$ & $8(100,0)$ & 741,83 & $122,00-1531,51$ \\
\hline & Total & 15 & 543,57 & $12(80,0)$ & 679,46 & $89,45-1592,21$ \\
\hline Total & & 36 & 321,59 & $26(72,2)$ & 445,28 & $89,45-1592,21$ \\
\hline
\end{tabular}

Letras diferentes na mesma coluna indicam diferença significativa segundo o teste de Kruskal-Wallis $(\mathrm{p}<0,05)$.

Limite de quantificação $=87,75 \mu \mathrm{g} / \mathrm{kg}$.

Limite máximo permitido para DON em grãos de trigo de $1750 \mu \mathrm{g} / \mathrm{kg}$ (COMUNIDADE EUROPÉIA, 2007) e 2000 $\mathrm{ug} / \mathrm{kg}$ em trigo integral a partir de 2012 (BRASIL, 2011).

Calori-Domingues et al. (2007) detectaram $94 \%$ de positividade em trigo brasileiro, com contaminação média $(332 \mu \mathrm{g} / \mathrm{kg})$, semelhante ao presente estudo, analisando 50 amostras provenientes de cooperativas ou empresas processadoras de São Paulo, Paraná e Rio Grande do Sul, enquanto em trigo proveniente da Argentina e Paraguai, detectaram níveis de 30 a $349 \mu \mathrm{g} / \mathrm{kg}$ (46\% de positividade, 50 amostras). Lamardo, Navas e Sabino (2006), analisando 28 amostras de trigo comercial no Estado de São Paulo, detectaram DON em 50 $\%$ de farinha, com média em amostras positivas de $296,3 \mu \mathrm{g} / \mathrm{kg}$, enquanto em trigo em grão com $33 \%$ de positividade, a média foi $753,2 \mu \mathrm{g} / \mathrm{kg}$, superior ao presente trabalho. Oliveira et al. (2002), analisando farinha de trigo e produtos de panificação comercializados em cidades de Minas Gerais, detectaram níveis de 40 a $1205 \mu \mathrm{g} / \mathrm{kg}$ em farinha, 78 a $780 \mu \mathrm{g} / \mathrm{kg}$ em pão de sal, 113 a $289 \mu \mathrm{g} / \mathrm{kg}$ em pão de forma e não-detectado em panetone. Pinto et al. (2008) detectaram $80 \%$ de positividade de DON em trigo na Argentina, com níveis superiores ao presente trabalho, variando de 300 a $70.000 \mu \mathrm{g} / \mathrm{kg}$, média de $9.873,33 \mu \mathrm{g} / \mathrm{kg}$.
Em contraste, Muthomi et al. (2008) detectaram níveis inferiores de DON em trigo produzido no Quênia, país de clima Tropical e baixa freqüência de F. graminearum, com valores variando de 105 a $303 \mu \mathrm{g} / \mathrm{kg}$ na safra de 2004 (48 amostras, positividade $75 \%$ ).

Um aumento de positividade e níveis de DON tem sido observado em trigo brasileiro nos últimos anos (OLIVEIRA et al., 2002; LAMARDO; NAVAS;SABINO, 2006; CALORI-DOMINGUES et al., 2007). Todavia, a contaminação por DON depende das condições meteorológicas, causando variações entre diferentes safras, devido a condições favoráveis ao desenvolvimento fúngico. Salienta-se a importância de contínuo monitoramento e desenvolvimento de métodos de fácil execução e elevada sensibilidade para detecção e quantificação de micotoxinas, para garantir a qualidade de alimentos.

A Tabela 3 mostra o consumo de pão francês e massas alimentícias e Ingestão Diária Estimada (IDE) de DON de acordo com cinco faixas etárias. Observou-se diferença de peso entre as faixas etárias, sendo que a faixa com idade entre 
31 e 40 anos, entre 41 e 50 anos e superior a 51 anos apresentaram peso mais elevado (dados não apresentados; $\mathrm{p}<0,05$ ). Com relação a quantidade consumida de pão, observou-se ingestão inferior pela faixa de idade superior a 51 anos $(\mathrm{p}<0,05)$, não sendo observada diferença significativa no consumo de massas alimentícias entre as faixas etárias (Tabela 3). A IDE de DON, considerando o consumo de pão francês pelos entrevistados, foi superior no grupo de 31 a 40 anos (média de $0,243 \mu \mathrm{g} / \mathrm{kg} / \mathrm{dia}$ ), enquanto IDE inferior ocorreu no grupo com mais de 51 anos, média de 0,125 $\mu \mathrm{g} / \mathrm{kg} / \mathrm{dia}(\mathrm{p}<0,05$, Tabela 3). A IDE de DON considerando consumo de massas alimentícias variou de 0,090 a $0,140 \mu \mathrm{g} / \mathrm{kg} / \mathrm{dia}$ entre os grupos etários, não havendo diferença significativa entre as faixas etárias ( $p>0,05$, Tabela 3$)$.

A Tabela 4 apresenta a IDE média de DON por meio do consumo de pão francês e massas alimentícias, comparando a estimativa obtida, considerando para o cálculo a contaminação global (Paraná e Rio Grande do Sul; média de DON de 333,78 $\mu \mathrm{g} / \mathrm{kg}$, total de 36 amostras, incluindo os valores não-detectados como $1 / 2$ LQ) versus amostras provenientes do Paraná (21 amostras, 177,66 $\mu \mathrm{g} / \mathrm{kg}$ ). Salienta-se que o Paraná é o maior produtor de trigo brasileiro, contribuindo com $53 \%$ de produção, assim como a coleta de dados de consumo foi conduzida em região que conta com diversas indústrias processadoras. No grupo considerando contaminação global $(333,78 \mu \mathrm{g} / \mathrm{kg})$, a Ingestão Diária Estimada (IDE) de DON variou de zero a 158,6 \% da Ingestão Diária Máxima Tolerável Provisória (IDMTP) para DON, sendo que dois indivíduos ultrapassaram a IDMTP de DON de 1 $\mu \mathrm{g} / \mathrm{kg}$ peso corporal/dia estabelecida pela JECFA (2001). O consumo de pão francês contribuiu com 18,7\% de IDMTP, enquanto o consumo de massas alimentícias contribuiu com 12,5\% de IDMTP. Os indivíduos classificados como "alto consumidor" (relataram consumir estes alimentos 2 vezes ao dia) apresentaram IDE de DON a partir de pão 2,7 vezes superior ao consumidor médio, e 7,1 vezes superior a partir de massas alimentícias. Direcionando o cálculo de IDE, considerando a contaminação das amostras do Paraná (média de $177,66 \mu \mathrm{g} / \mathrm{kg}$ ), nenhum indivíduo ultrapassou a IDMTP, com valores variando de $6,6 \%$ a 84,4 $\%$. A IDE, considerando contaminação global, foi 1,8 vezes superiores ao valor considerando as amostras parananeses $(\mathrm{p}<0,05)$.

Tabela 3. Ingestão Diária Estimada de desoxinivalenol (DON) por meio do consumo de pão francês e massas alimentícias considerando diferentes faixas etárias em Londrina-PR.

\begin{tabular}{|c|c|c|c|c|c|c|c|c|}
\hline \multirow[t]{4}{*}{ Faixa etária } & \multirow[t]{4}{*}{$\mathbf{N}$} & \multirow{2}{*}{\multicolumn{2}{|c|}{$\begin{array}{l}\text { Consumo } \\
\text { (g/semana) }\end{array}$}} & \multicolumn{4}{|c|}{ Ingestão Diária Estimada de DON ( $\mu \mathrm{g} / \mathrm{kg}$ p.c./dia) } & \multirow[t]{4}{*}{$\%$ IDMTP } \\
\hline & & & & \multicolumn{2}{|c|}{ Pão francês } & \multicolumn{2}{|c|}{ Massas alimentícias } & \\
\hline & & Pão & Massas & Média & Faixa & Média & Faixa & \\
\hline & & \multicolumn{6}{|c|}{ francês alimentícias } & \\
\hline$<20$ anos & 55 & $285,5^{\mathrm{a}}$ & $156,2^{\mathrm{a}}$ & $0,225^{\mathrm{a}, \mathrm{b}}$ & $0,0-0,931$ & $0,140^{\mathrm{a}}$ & $0,0-1,128$ & 36,5 \\
\hline $21-30$ anos & 103 & $245,5^{\mathrm{a}}$ & $154,2^{\mathrm{a}}$ & $0,171^{\mathrm{a}, \mathrm{b}}$ & $0,0-0,727$ & $0,130^{\mathrm{a}}$ & $0,0-0,611$ & 29,9 \\
\hline $31-40$ anos & 38 & $361,5^{\mathrm{a}}$ & $107,9^{\mathrm{a}}$ & $0,243^{\mathrm{a}}$ & $0,0-0,573$ & $0,090^{\mathrm{a}}$ & $0,0-0,296$ & 33,0 \\
\hline $41-50$ anos & 31 & $273,4^{\mathrm{a}}$ & $150,9^{\mathrm{a}}$ & $0,172^{a, b}$ & $0,0-0,677$ & $0,110^{\mathrm{a}}$ & $0,0-0,509$ & 27,5 \\
\hline$>51$ anos & 34 & $190,1^{\mathrm{a}}$ & $185,2^{\mathrm{a}}$ & $0,125^{b}$ & $0,0-0,497$ & $0,136^{\mathrm{a}}$ & $0,0-0,905$ & 25,0 \\
\hline
\end{tabular}

Total de indivíduos entrevistados $=260$. Total de habitantes $=447.000$.

Letras diferentes na mesma coluna indicam diferença significativa segundo o teste de Kruskal-Wallis ( $\mathrm{p}<0,05)$.

\% IDMTP= porcentagem de Ingestão Diária Estimada em relação à IDMTP (Ingestão Diária Máxima Tolerável Provisória $=1 \mu \mathrm{g} / \mathrm{kg}$ p.c./dia). 
Tabela 4. Ingestão Diária Estimada de desoxinivalenol (DON) considerando consumo de pão francês e massas alimentícias em Londrina-PR.

\begin{tabular}{|c|c|c|c|c|c|c|}
\hline \multirow[t]{3}{*}{ Alimento } & \multicolumn{6}{|c|}{ Ingestão Diária Estimada de DON ( $\mu \mathrm{g} / \mathrm{kg}$ p.c./dia) } \\
\hline & \multicolumn{3}{|c|}{ Todas as amostras* } & \multicolumn{3}{|c|}{ Amostras do Paraná** } \\
\hline & Média & Faixa & Alto consumidor $* * *$ & Média & Faixa & Alto consumidor $* * *$ \\
\hline Pão & 0,187 & $0,0-0,931$ & 0,502 & 0,100 & $0,0-0,495$ & 0,267 \\
\hline francês & & & $(0,313-0,931)$ & & & $(0,167-0,495)$ \\
\hline Massas & 0,125 & $0,0-1,128$ & 0,881 & 0,066 & $0,0-0,600$ & 0,469 \\
\hline alimentícias & & & $(0,611-1,128)$ & & & $(0,325-0,600)$ \\
\hline Total & $0,308^{\mathrm{a}}$ & $0,0-1,586$ & & $0,168^{\mathrm{b}}$ & & $0,006-0,844$ \\
\hline
\end{tabular}

* Cálculo de IDE considerando contaminação média de DON de 333,78 $\mu \mathrm{g} / \mathrm{kg}$ (análise de 36 amostras provenientes de Rio Grande do Sul e Paraná).

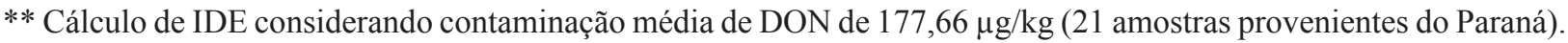

*** Indivíduo que relatou consumir o alimento 2 vezes ao dia.

Letras diferentes na mesma linha indicam diferença significativa, segundo o teste de Mann-Whitney $(\mathrm{p}<0,05)$.

Salienta-se que o pão francês e as massas alimentícias não são os únicos alimentos responsáveis pela ingestão de DON na dieta, embora sejam os maiores contribuintes. Os relatos também apontaram que altos consumidores excederam a IDMTP na França e Alemanha, conforme dados da ocorrência de toxinas de Fusarium spp. e avaliação da ingestão diária pela população Européia (SCHOTHORST; VAN EGMOND, 2004). Turner et al. (2008) analisando urina de 300 indivíduos no Reino Unido, obtiveram positividade de 98,7 \% de DON, com média geométrica de 9,42 $\mu \mathrm{g} /$ dia (ND$65,97 \mu \mathrm{g} / \mathrm{dia}$ ), sendo os cereais mais consumidos o pão, as massas alimentícias, os bolos e os cereais matinais, com nível de DON urinário associado a quantidade consumida $(p<0,0005)$. Embora IDE tenha sido $0,319 \mu \mathrm{g} / \mathrm{kg}$ p.c./dia naquele trabalho, 4 indivíduos $(1,3 \%)$ ultrapassaram a IDMTP, sendo este valor superior ao nosso trabalho $(0,308 \mu \mathrm{g} /$ kg p.c./dia, Tabela 4). As Tabelas 3 e 4 apresentam a avaliação inicial de IDE de DON por meio dos derivados de trigo no país, estando em andamento estudo englobando maior amostragem.

O contínuo monitoramento de DON em grãos in natura, bem como o efeito de processamento industrial em resíduos tóxicos constituem assuntos prioritários na segurança de alimentos. Além disso, a regulamentação governamental quanto aos níveis de micotoxinas abrangendo maior número de produtos agrícolas é necessária, a fim de promover a melhoria no abastecimento de alimentos seguros.

\section{Conclusão}

Os níveis de DON em trigo cultivado nos Estados do Paraná e Rio Grande do Sul apresentaramse abaixo do limite máximo estabelecido pela Comunidade Européia para trigo não-processado, indicando a segurança dos produtos derivados. Pão francês e massas alimentícias foram os principais derivados consumidos em LondrinaPR. Considerando o consumo de pão e massas alimentícias, dois indivíduos $(0,8 \%)$ ultrapassaram a Ingestão Diária Máxima Tolerável Provisória (IDMTP).

\section{Agradecimentos}

Ao Conselho Nacional de Desenvolvimento Científico e Tecnológico-CNPq, CNPq/MAPA, 
CAPES, UGF-Fundo Paraná da SETI-PR e Fundação Araucária pelo apoio financeiro, assim como a CNPq, CAPES e Fundação Araucária pela bolsa aos Pós-graduandos, Iniciação Científica e Produtividade aos integrantes do grupo. Ao Depto de Estatística e a Prof ${ }^{\mathrm{a}} \mathrm{Dr}^{\mathrm{a}}$ Inês Cristina de Batista Fonseca da Universidade Estadual de Londrina pela assessoria estatística concedida.

\section{Comitê de Ética em Pesquisa}

A pesquisa referente ao consumo de produtos derivados de trigo foi autorizada pelo 'Comitê de Ética em Pesquisa Envolvendo Seres Humanos' da Universidade Estadual de Londrina, através de Parecer $n^{\circ} 173 / 07$. Realizada de acordo com as normas técnicas de segurança e bioética.

\section{Referências}

AYRES, M.; AYRES JÚNIOR, M.; AYRES, D. L.; SANTOS, A. A. BIOESTAT - Aplicações estatísticas nas áreas das ciências Bio-Médicas. Ong Mamiraua. Belém, PA, 2007.

BRASIL. Agência Nacional de Vigilância Sanitária. Resolução RDC no 7 de 18 de fevereiro de 2011, DOU de 22/02/2011. Dispõe sobre limites máximos tolerados (LMT) para micotoxinas em alimentos, 2011.

Resolução RDC $n^{\circ} 359$ de 23 de dezembro de 2003. Aprova o Regulamento Técnico de Porções de Alimentos Embalados para Fins de Rotulagem Nutricional, 2003.

- Ministério da Agricultura, Pecuária e Abastecimento. Instrução Normativa $N^{o} 7$ de 15 de agosto de 2001. Aprova o Regulamento Técnico de Identidade e de Qualidade do Trigo.

BRIONES-REYES， D.; GOMÉZ-MARTINEZ， L.; CUERVA-ROLÓN, R. Zearalenone contamination in corn for human consumption in the state of Tlaxcala, México. Food Chemistry, Kidlington, v. 100, n. 2, p. 693-698, 2007.

CALORI-DOMINGUES, M. A.; ALEMIDA, R. R.; TOMIWAKA, M. M.; GALLO, C. R.; GLORIA, E. M.; DIAS, C. T. S. Ocorrência de desoxinivalenol em trigo nacional e importado utilizado no Brasil. Ciência e Tecnologia de Alimentos, Campinas, v. 27, n. 1, p. 181185, 2007.
COMMISSION OF THE EUROPEAN COMMUNITIES. Commission Regulation (EC) $N^{o}$ 1126/2007 of 28 September 2007, amending Regulation (EC) $N^{\circ} 1881 / 2006$ setting maximum levels for certain contaminants in foodstuffs as regards Fusarium toxins in maize and maize products. [S.1 : s.n], 2007.

EMBRAPA. EMPRESA BRASILEIRA DE PESQUISA AGROPECUARIA - EMBRAPA. Panorama mundial do trigo no Fórum Nacional. 2010. Disponível em: <www. embrapa.br>. Acesso em: 10 jul. 2010.

FUJITA, A. H.; FIGUEROA, M. O. R. Composição centesimal e teor de $\beta$-glucanas em cereais e derivados. Ciência e Tecnologia de Alimentos, Campinas, v. 23, n. 2, p. 116-120, 2003.

INSTITUTO ADOLFO LUTZ - IAL. Métodos físicoquímicos para análise de alimentos. Coordenadores Odair Zenebon, Neus Sadocco Pascuet e Paulo Tiglea. São Paulo: Instituto Adolfo Lutz, 2008, 1020 p.

INSTITUTO BRASILEIRO DE GEOGRAFIA E ESTATÍSTICA - IBGE. 2009. Disponível em <www. ibge.gov.br>. Acesso em: 30 ago. 2009.

INTERNATIONAL AGENCY FOR RESEARCH ON CANCER - IARC. Monographs on the evaluation of carcinogenic risks to humans. v. 56: Some naturally occurring substances, food items and constituents, heterocyclic aromatic amines and mycotoxins. World Health Organization, 1993. p. 397-333.

INTERNATIONAL PROGRAMME ON CHEMICAL SAFETY - IPCS/GEMS. Food Euro workshop on reliable evaluation of low level contamination of food, Appendix 5, Kulmbach, Germany, May 1995.

JOINT FAO/WHO EXPERT COMMITTEE ON FOOD ADDITIVES - JECFA. Safety evaluation of certain mycotoxins in food, prepared by the fifty-sixth Meeting of the Joint FAO/WHO Expert Commitee on Food Additives, WHO Food Additives Series 47 (WHO Geneva). 2001.

KUIPER-GOODMAN, T. Risk assessment of mycotoxins. In: A collection of invited papers presented at the Seventh International IUPAC Symposium on Mycotoxins and Phycotoxins. p. 257-264, 1988.

LAMARDO, L. C. A.; NAVAS, S.; SABINO, M. Desoxinivalenol (DON) em trigo e farinha de trigo comercializados na cidade de São Paulo. Revista do Instituto Adolfo Lutz, São Paulo, v. 65, n. 1, p. 32-35, 2006.

MUTHOMI, J. W.; NDUNG'U, J. K.; GATHUMBI, J. K.; MUTITU, E. W.; WAGACHA, J. M. The occurrence of Fusarium and mycotoxins in Kenyan wheat. Crop Protection, Oxford, v. 27, n. 8, p. 1215-1219, 2008. 
OLIVEIRA, M. S.; PRADO, G.; ABRANTES, F. M.; SANTOS, L. G.; VELOSO, T. Incidência de aflatoxinas, desoxivalenol e zearalenona em produtos comercializados em cidades do Estado de Minas Gerais no período de 1998 - 2000. Revista do Instituto Adolfo Lutz, São Paulo, v. 61 , n. 1, p. 1-6, 2002.

ONO, E. Y. S.; BIAZON, L.; SILVA, M.; VIZONI, E.; SUGIURA, Y.; UENO, Y.; HIROOKA, E. Fumonisins in corn: correlation with Fusarium sp. count, damaged kernels, protein and lipid content. Brazilian Archives of Biology and Technology, Curitiba, v. 49, n. 1, p. 63-71, 2006.

ONO, E. Y. S.; SASAKI, E. Y.; HASHIMOTO, E. H.; HARA, L. N.; CORREA, B.; ITANO, E. N.; SUGIURA, T.; UENO, Y.; HIROOKA, E. Y. Post-harvest storage of corn: effect of beginning moisture content on mycoflora and fumonisin contamination. Food Additives and Contaminants, New York, v. 19, n. 11, p. 1081-1090. 2002.

PINTO, V. E. F.; TERMINIELLLO, L. A.; BASILICO, J. C.; RITIENI, A. Natural occurrence of nivalenol and mycotoxigenic potential of Fusarium graminearum strains in wheat affected by head blight in Argentina. Brazilian Journal of Microbiology, São Paulo, v. 39, n. 1, p. 157-162, 2008.

PRANGE, A.; BIRZELE, B.; KRÄMER, J.; MEIER, A.; MODROW, H.; KÖHLER, P. Fusarium-inoculated wheat: deoxynivalenol contents and baking quality in relation to infection time. Food Control, Amsterdam, v. 16, n. 8, p. 739-745, 2005.

RAMIREZ, M. L.; CHULZE, S.; MAGAN, N. Temperature and water activity effects on growth and temporal deoxynivalenol production by two Argentinean strains of Fusarium graminearum on irradiated wheat grain. International Journal of Food Microbiology, Amsterdam, v. 106, n. 3, p. 291-296, 2006.

SANTOS, J. S.; OLIVEIRA, T. M.; ALMEIDA-FILHO, J. T. P.; ONO, E. Y. S.; HIROOKA, E. Y. Avaliação da ingestão de derivados de trigo em Londrina, PR, por meio de questionário de frequência de consumo de alimentos. Nutrire (SBAN), São Paulo, v. 34, n. 3, p. 153-164, 2009.

SCHOTHORST, R. C.; VAN EGMOND, H. P. Report from SCOOP task 3.2.10 "collection of occurrence data of Fusarium toxins in food and assessment of dietary intake by the population of EU member states". Toxicology Letters, Amsterdam, v. 153, n. 1, p. 133-143, 2004.

STEYN, P. S. Mycotoxins, general view, chemistry and structure. Toxicology Letters, Amsterdam, v. 82/83, p. 843-851, 1995.

TURNER, P. C.; ROTHWELL, J. A.; WHITE, K.
L. M.; GONG, Y.; CADE, J. E.; WILD, C. P. Urinary deoxynivalenol is correlated with cereal intake in individuals from the United Kingdom. Environmental Health Perspectives, North Carolina, v. 16, n. 1, p. 21$25,2008$.

VIEIRA, A. P.; BADIALE-FURLONG, E.; OLIVEIRA, M. L. M. Ocorrência de micotoxinas e características físico-químicas em farinhas comerciais. Ciência $e$ Tecnologia de Alimentos, Campinas, v. 19, n. 2, p. 221225, 1999. 